\title{
Microvascular myocutaneous and cutaneous free flap reconstruction in patients with terminal esophagostomy after complicated oncological esophagus resection
}

\author{
Lukas Poelsler (D) - Oliver Koch · Alexander Gaggl · Jaroslav Presl · Joerg Hutter · Christian Brandtner · \\ Klaus Emmanuel
}

Received: 6 November 2021 / Accepted: 15 November 2021 / Published online: 23 December 2021

(C) The Author(s) 2021

\begin{abstract}
Summary
Background Persisting anastomotic leakage after oncological esophagectomy is a severe problem associated with high mortality and morbidity. Unfortunately, treatment options with promising results are scarce, especially when conventional operative and endoscopic methods have failed. Due to limitation of oral intake and the need for artificial nutrition, quality of life (QOL) is reduced. Microvascular myocutaneous and cutaneous free flap (MFF) reconstruction could be a promising alternative.

Methods This retrospective case series presents $7 \mathrm{pa}-$ tients treated between March 2017 and November 2020 at our surgical department, with persisting postoperative anastomotic leakage without further feasible treatment options. All patients received anastomotic MFF reconstruction; used flaps and important intraoperative factors like microvascular anastomosis were evaluated. Complications and patient contentment was assessed.

Results The included 7 male patients had median age of 65.15 years (range: 48-75). MFF function was adequate in 6/7 patients, one flap necrosis was encountered. Whereas 5 patients initially had good results, surgical revision was performed in 1 patient to ensure graft function. Postoperative complications appeared in 6/7 patients. Mean duration of inpatient care was 63 days (range: 24-156). At the time of evaluation, 1 patient has died of his malignant disease. No more
\end{abstract}

\footnotetext{
L. Poelsler $\cdot$ O. Koch, MD $(\bowtie) \cdot$ J. Presl $\cdot$ J. Hutter $\cdot$

K. Emmanuel

Department of Surgery, Paracelsus Medical University,

Muellner Hauptstraße 48, 5020 Salzburg, Austria

o.koch@salk.at
}

A. Gaggl · C. Brandtner

Department of Oral and Maxillofacial Surgery, Paracelsus

Medical University Salzburg, Salzburg, Austria additional nutrition was needed in 3/6 patients with adequate graft function. Most patients reported improved QOL after MFF.

Conclusion MFF free flap can be an alternative treatment option for patients with terminal esophagostomy after complicated oncological esophagus resection without further treatment options. The renewed ability of oral food intake results in a significant improvement of QOL.

Keywords Esophageal cancer - Microvascular free flap reconstruction · Anastomotic leak · Upper gastrointestinal surgery · Cervical esophagostomy

\section{Background}

Esophageal cancer remains a rare diagnosis with limited prognosis in the Western world. In Austria, 423 patients were diagnosed with esophageal cancer in 2018. Recently published numbers of the Austrian national registry of esophageal resection compare well with results of renowned international centers, with major complications appearing in $21.7 \%$ and a mortality rate of $2.9 \%$ [1, 2]. Squamous cell carcinoma (SCC) and adenocarcinoma are the most frequently observed types of esophageal cancer, with 5.2 per 100,000 for SCC and 0.7 per 100,000 for adenocarcinoma. SCC is most frequently observed in Asia, whereas adenocarcinoma is more frequently observed in Europe, with a rising incidence compared to SCC in recent years [3]. Medication-based therapies for oncological diseases have rapidly improved over the years [4]. However, surgical esophagus resection is considered the gold standard treatment and the only potentially curative treatment option. Locally advanced tumors should receive neoadjuvant therapy to lower locoregional recurrence rates and to ensure R0 resection. Widely used treatment options are 
neoadjuvant chemotherapies as well as neoadjuvant chemoradiation. In Europe, the currently most commonly used neoadjuvant chemoradiation scheme is CROSS. Preoperative radiation is known to be an independent risk factor for local complications; complications appear more often, especially when radiation dosage increases. Spread in the lymph nodes has been shown to be a risk factor for recurrence after initial treatment. Therefore, decision-making is difficult, especially in early-stage esophageal cancer, because in lesions classified as T1, endoscopic treatment is feasible. Tumors with superficial or submucosal involvement can be treated with endoscopic resection, but tumors with deep submucosal involvement should be treated with surgical esophagectomy because lymph node involvement is significantly higher [5-8].

Typically, esophagus resection is followed by gastric tube pull-up reconstruction, either anatomical or extraanatomical retrosternal pull-up. SCC usually requires McKeown esophagectomy with cervical anastomosis, and adenocarcinoma can more often be treated by Ivor-Lewis esophagectomy with intrathoracic anastomosis. Minimally invasive operation techniques have improved patient outcome lately, but esophagus resection remains a procedure with high mortality and morbidity. Especially anastomosis insufficiency remains a major problem. In patients with SCC who received cervical anastomosis, prognosis depends on limitation to the cervical region or further intrathoracic manifestation if insufficiency appears. Thoracic manifestation is associated with higher mortality and morbidity. The major reason for persisting anastomotic leakage is gastric pull-up tube necrosis. This complication is rare, appearing in approximately $1 \%$ of esophagus resections, with gastric pull-up leading to necrosis of the gastric conduit with need for surgical revision [9]. Earlier there was no standardized classification of anastomotic insufficiency after esophagectomy established. In 2015, a consensus guideline (CAES classification) for complication assessment was published. It has been shown that the CAES classification is valid and, therefore, it should be clinically applied in all centers $[10,11]$. Further used reconstruction techniques are colonic interposition or interposition of small bowel. Colonic interposition requires more anastomosis, which increases the risk of anastomotic leak. Therefore, it is less frequently used [12]. However, treatment options are scarce if secondary interposition develops necrosis as well or fistulation occurs, and terminal esophagostomy is sometimes the only solution $[13,14]$. This condition is associated with poor quality of life due to the impossibility of oral intake. Quality of life is most impacted by the possibility of oral nutrition-even in jejunum interposition, quality of life is described to improve significantly [15]. Need for in-hospital-stay is frequent and therefore associated with high costs.

Despite multiple reconstructive approaches proposed in the past, there remains no consensus regard- ing the optimal method for reconstructing a terminal esophagostomy $[6,14,16,17]$, especially in patients after a complicated postoperative course who received preoperative radiotherapy and are without treatment options. Microvascular myocutaneous and cutaneous free flap reconstruction could be a promising alternative for such patients. The use for esophageal reconstruction has been described previously [17]. The aim of this paper is to analyze the outcome of microvascular myocutaneous and cutaneous free flap reconstruction in patients with terminal esophagostomy. Additionally, patients with persisting cervical anastomotic leak after complicated oncological esophagus resection with MFF reconstruction were included.

\section{Patients and methods}

We performed a retrospective analysis of all patients who received MFF reconstruction between March 2017 and November 2020 at the University Hospital Salzburg in cooperation with the Department of Surgery and the Department of Oral and Maxillofacial Surgery. During the study period, 7 patients were treated. All patients who received MFF reconstruction were male; 6 patients initially received oncological esophagus resection because of SCC, 1 patient initially received hypopharyngeal resection after an SCC diagnosis followed by esophageal resection because of esophageal recurrence. Gastric pull-up was performed in all 7 patients. Secondary carcinoma was diagnosed in 3 out of 7 patients $(42.8 \%)$ at the staging procedure, with hypopharynx being the most common location. Postoperatively, all patients developed severe complications. After exhausting all different treatment options salivary stomata was applied in all patients. Regarding CAES classification, all patients were classified as stage IIIb. Endoscopic therapies like vacuum based treatment options, endoscopic dilatation, and continuous endoscopic monitoring over a short period of time were performed. Additionally, 4 patients received colon interposition. Furthermore, 1 patient received jejunum interposition after developing necrosis of colonic interposition. Endoscopic and open vacuum pressure therapy (VAC) were performed in 6 patients $(85.7 \%)$. Indication for primary MFF replacement after gastric pull-up necrosis was imposed in 2 patients because of tracheal fistulation.

Mean age of the patients was 65.15 years (range: 48-75 years). Mean preoperative weight was $59.86 \mathrm{~kg}$ (range: $49-74 \mathrm{~kg}$ ) with a mean height of $175.14 \mathrm{~cm}$ (range: $160-183 \mathrm{~cm}$ ). Body mass index (BMI) was evaluated: mean BMI was $19.45 \mathrm{~kg} / \mathrm{m}^{2}$ (range: 16$23.36 \mathrm{~kg} / \mathrm{m}^{2}$ ). Before resection of the primary, 6 out of 7 patients $(85.7 \%)$ received neoadjuvant radiation. Gray dose was available in 4 out of 6 patients $(66.7 \%)$. Mean Gray dose was 58.075 Gy (range: 41.4-66.0 Gy). TNM-LVR classification, grading, and UICC stage are reported in Table 1. T3 stadium was most frequently 
Table 1 TNM-LVR classification, UICC stage, and grading of primary tumors

\begin{tabular}{l|ll}
\hline Classifications & Number of patients & $\%$ \\
\hline T stage & 1 & 14.3 \\
\hline Tis & 1 & 14.3 \\
\hline T1b & 1 & 14.3 \\
\hline T2 & 3 & 42.8 \\
\hline T3 & & \\
\hline N stage & 4 & 57.1 \\
\hline N0 & 2 & 28.6 \\
\hline N1 & & \\
\hline M stage & 6 & 85.7 \\
\hline M0 & 6 & 85.7 \\
\hline L stage & & \\
\hline L0 & 6 & 85.7 \\
\hline V stage & 5 & \\
\hline V0 & 1 & 71.4 \\
\hline R stage & 1 & 14.3 \\
\hline R0 & 1 & 14.3 \\
\hline R1 & 1 & 14.3 \\
\hline Stage grouping according & UICC & 14.3 \\
\hline 0 & 1 & 14.3 \\
\hline IA & 2 & 28.6 \\
\hline IB & 3 & 42.8 \\
\hline IIA & 2 & 28.6 \\
\hline IIIA & & \\
\hline Grading & & \\
\hline G2 & & \\
\hline G3 & & \\
\hline & & \\
\hline
\end{tabular}

observed. Complete data were available in 6 out of 7 patients. All patients received reconstruction with microvascular myocutaneous and cutaneous free flap, the used free flaps are reported in Table 2. The flaps were used to restore continuity of oral cavity and esophagus. This was achieved by harvesting the flap at first with sufficient length of appertain vessels (e.g., Fig. 1a and 2a). After successful checking for sufficient perfusion, the flap was transferred to the neck (e.g., Fig. 1c and 2d). The flap was disposed in a circular form (e.g., Fig. 1c and 2b) and then connected to gastric conduit or colonic interposition. The used vessels for microvascular anastomosis are reported in Table 3.

Table 2 Overview of used free flaps

\begin{tabular}{|c|c|}
\hline $\begin{array}{l}\text { Type of microvascular myocutaneous and cutaneous } \\
\text { free flap }\end{array}$ & $\begin{array}{l}\text { Number of uses } \\
(\%)\end{array}$ \\
\hline Anterior lateral thigh free flap (ALT) & $4(57.1)$ \\
\hline Radialis free flap & $1(14.3)$ \\
\hline $\begin{array}{l}\text { Osteomyocutaneous free flap from medial femoral } \\
\text { condyle }\end{array}$ & $1(14.3)$ \\
\hline Left brachial free flap & $1(14.3)$ \\
\hline
\end{tabular}

Table 3 Used vessels for microvascular anastomosis

\begin{tabular}{ll} 
Vessel for microvascular anastomosis & Number of uses (\%) \\
\hline Superior thyroid artery & $5(71.4)$ \\
\hline Facial artery & $1(14.3)$ \\
\hline Transverse cervical artery & $1(14.3)$
\end{tabular}

\section{Complications and patient contentment}

Complications were obtained according to the Clavien-Dindo classification. Additionally, we evaluated patient contentment after the surgical procedure with three questions ("Are you able to swallow appropriately?" "Has your quality of life improved, stayed steady, or worsened since the operation was performed?" "With the knowledge now, would you consent to this procedure again?").

\section{Results}

\section{Graft results}

MFF function was adequate in 6 out of 7 patients (85.7\%); flap necrosis appeared in 1 patient (14.3\%). Flap results were initially good in 5 patients, 1 more patient needed surgical revision to ensure graft function. No local complications appeared after free flap mobilization. Speaking was not feasible in either patient with tracheal fistulation: 1 patient regained speaking ability, 1 patient can speak with an appropriate tracheal canula. The patient with an osteomyocutaneous free flap from the medial femoral condyle developed a satisfactory result: no complications occurred and full speaking ability was regained by the patient. The lateral arm flap was rejected, yet this graft choice can be applied.

\section{Complications and patient contentment}

Of 6 patients, 4 reported improved quality of life. Results of the three questions are reported in Table 4. No more additional artificial nutrition was needed in 3 out of 6 patients (50\%). Of the 6 patients, 5 would

Table 4 Three questions to assess patient contentment

\begin{tabular}{|c|c|c|}
\hline Asked questions & Possible answers & $\begin{array}{l}\text { Number of answers } \\
(n=6)\end{array}$ \\
\hline \multirow{3}{*}{$\begin{array}{l}\text { Question 1: Are you able } \\
\text { to swallow appropriately? }\end{array}$} & Yes, even solid food & $3(50 \%)$ \\
\hline & $\begin{array}{l}\text { Yes, but with cutaneous } \\
\text { fistulation }\end{array}$ & $1(16.7 \%)$ \\
\hline & No & $2(33.3 \%)$ \\
\hline \multirow{4}{*}{$\begin{array}{l}\text { Question 2: Has your } \\
\text { quality of life improved, } \\
\text { stayed steady, or wors- } \\
\text { ened since the operation } \\
\text { was performed? }\end{array}$} & Massive improvement & $2(33.3 \%)$ \\
\hline & Improvement & $1(16.7 \%)$ \\
\hline & Steady & $2(33.3 \%)$ \\
\hline & Worse & $1(16.7 \%)$ \\
\hline \multirow{2}{*}{$\begin{array}{l}\text { With the knowledge now, } \\
\text { would you consent to this } \\
\text { procedure again? }\end{array}$} & Yes & $5(83.3 \%)$ \\
\hline & No & $1(16.7 \%)$ \\
\hline
\end{tabular}


Fig. 1 Intraoperative pictures of anterior lateral thigh free flap (ALT) reconstruction. a harvesting of ALT free flap, b vessel preparation, c placement of ALT as interposition to ensure esophagus continuity with placed gastric tube, d finished placement of ALT with newly functionating esophagus
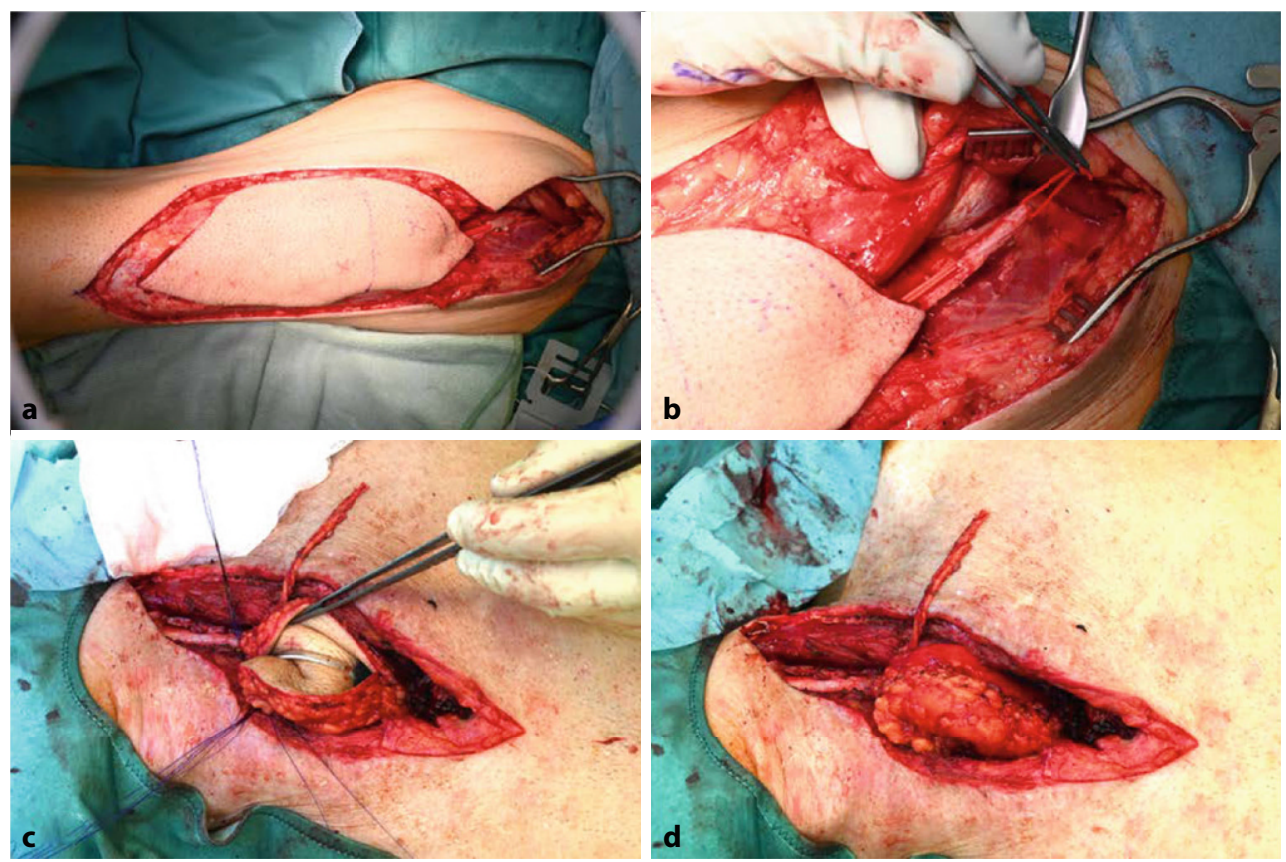

Fig. 2 Intraoperative pictures of lateral brachial free flap. a harvesting of free flap, b circular formation of free flap, c preparations for free flap interposition, d placement of circular free flap with gastric tube
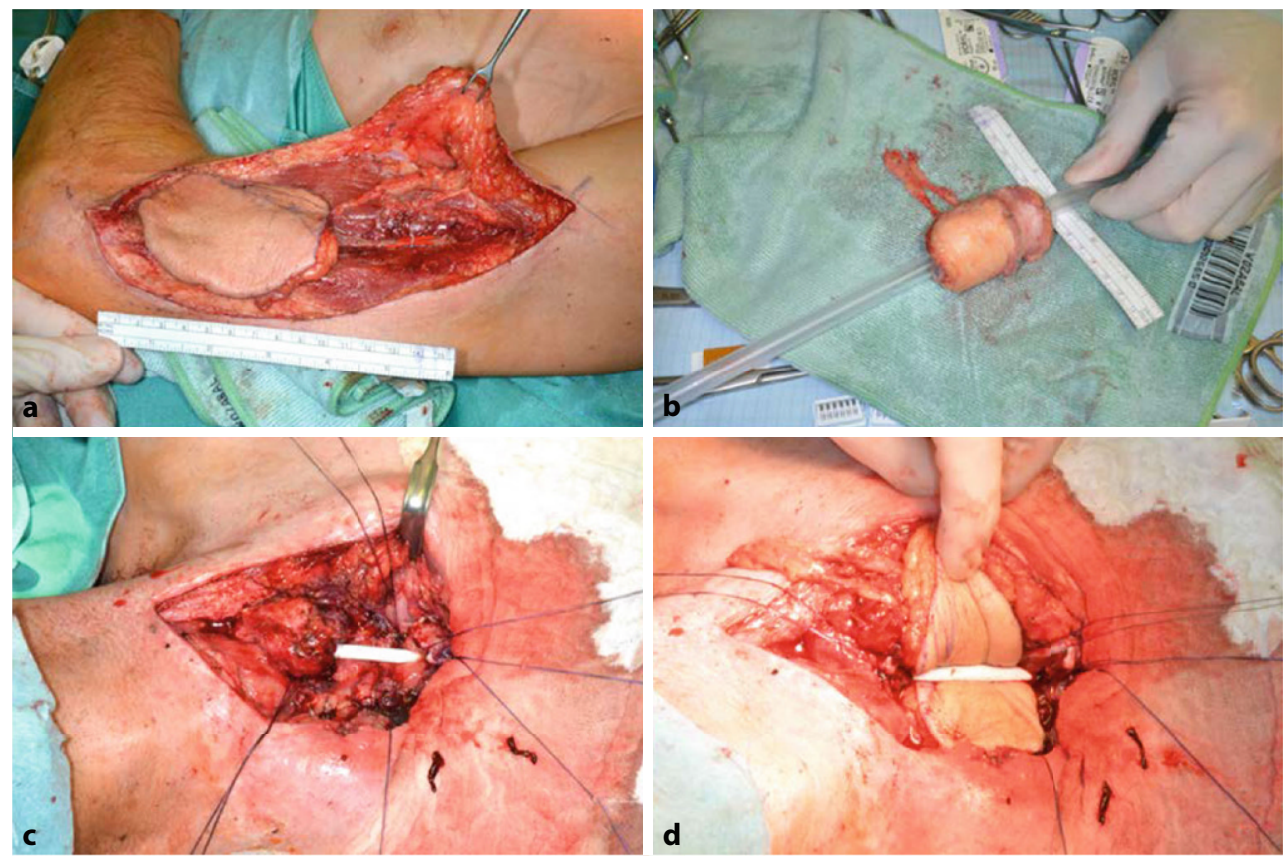

consent to this procedure again. Postoperative complications appeared in 6 out of 7 patients: 3 patients had complications ranked as Clavien-Dindo IIIa (2 patients received stenting, 1 patient endoscopic stenting) and 2 patients needed surgical revision (Clavien-Dindo IIIb). Furthermore, 1 patient had postoperative delirium with prolonged intensive care unit stay and 1 patient had no complications (Clavien-Dindo 0). Mean duration of inpatient care was 63 days (range: $24-156$ ). At the time of evaluation, one patient had died of his malignant disease.

\section{Discussion}

The results of this paper shows that MFF free flap is a safe and feasible treatment option for patients with terminal esophagostomy after complicated oncological esophagus resection. There was no procedure-related mortality observed and only 1 patient reported worsening quality of life, while patients with sufficient graft function reported massive regain of quality of life. Therefore, especially due to the high subjective level of suffering and low quality of life, this procedure can be considered. Despite the occurring complications and insufficient results in some patients, 
all patients besides one would consent to this treatment option again. This implicates the subjective poor quality of life prior to surgery. The number of patients who regained the ability of oral food intake was encouraging, especially in consideration of the few other available treatment options with the permanent need for cervical esophagostomy. Adequate graft function resulted in a significant improvement of quality of life for the patients. Only one patient reported lower quality of life than before free flap reconstruction, unfortunately this patient had a progressive disease after reconstructive surgery. Anterior lateral thigh (ALT) free flap reconstruction has been a frequently used free flap reconstruction technique for head and neck reconstructions since its initial description in 1983 [18]. However, free flaps with vascularized bone components are described to deliver better results due to a lower risk of reabsorption. Free flap harvesting from the medial knee has been performed since the early 1990s, and can be used for reconstruction of postoperative defects, especially after tumor resection. However, vascular anatomy may diverge. Therefore, routine preoperative vascular imaging is required before flap harvesting [19, 20]. Radial forearm free flaps are also commonly used for reconstructions in the head and neck area. Still, free flap failure is a scarce but occurring problem. Graft failure is differentiated into early and late graft failure, with failure after the seventh postoperative day considered as late failure. The most important risk factor for graft failure is preoperative irradiation. The most frequently observed reasons for late graft failure are abscess formation and vascular compromise. Careful graft observation is recommended within the first 14 postoperative days, to detect graft dysfunction early $[21,22]$. Unfortunately, one graft was initially rejected in our group. Due to the small size of the total sample, some grafts were only used once. Results of the used grafts does therefore not necessarily implicate general outcome in larger cohorts. Flaps for reconstruction should be selected individually and mutually with the patient. Rejection of graft remains a rare but occurring problem, with few possible preventions. Treatment options are exhausted in patients with multiple failed reconstruction techniques after esophagus resection. Therefore, every further applied strategy needs to be well considered and discussed with the patient. MFF reconstruction offers an additional treatment option for those patients. However, this procedure has high risks, with numerous possible complications. Patients eligible for this treatment should be selected carefully and perioperative risks have to be reduced preoperatively, for example by adequate monitoring of diabetes and sufficient hemoglobin levels.

This study has potential limitations. We performed MFF reconstruction on a rather small patient sample. Furthermore, this study was performed as a retrospective cohort study, which are known for limitations. Within this study the analysis of quality of life could only be performed retrospectively. Additionally available data of patients primarily treated extramurally was not completely available.

However, the outcome of patients after MFF reconstruction in this paper shows that the method might be a relevant treatment option to be discussed with patients if expertise in free flap reconstruction and esophageal surgery are available at the primary treatment center.

In conclusion, MFF reconstruction can be an alternative treatment option in patients with complicated postoperative courses with failure of primary reconstruction. MFF reconstruction has its role in patients with special defects like tracheal fistulation or as an option after conventional strategies have failed. Due to exhaustion of therapeutic strategies, newly applied strategies need to be discussed in detail, including all beneficial and possible adverse aspects, with the patient. For patients without further feasible treatment options, MFF reconstruction is a promising alternative to improve quality of life.

Funding Open access funding provided by Paracelsus Medical University.

\section{Declarations}

Conflict of interest L. Poelsler, O. Koch, A. Gaggl, J. Presl, J. Hutter, C. Brandtner, and K. Emmanuel declare that they have no competing interests.

Ethical standards All procedures performed in studies involving human participants or on human tissue were in accordance with the ethical standards of the institutional and national research committee and with the 1975 Helsinki declaration and its later amendments or comparable ethical standards. Informed consent was obtained from all individual participants included in the study.

Open Access This article is licensed under a Creative Commons Attribution 4.0 International License, which permits use, sharing, adaptation, distribution and reproduction in any medium or format, as long as you give appropriate credit to the original author(s) and the source, provide a link to the Creative Commons licence, and indicate if changes were made. The images or other third party material in this article are included in the article's Creative Commons licence, unless indicated otherwise in a credit line to the material. If material is not included in the article's Creative Commons licence and your intended use is not permitted by statutory regulation or exceeds the permitted use, you will need to obtain permission directly from the copyright holder. To view a copy of this licence, visit http://creativecommons.org/licenses/by/4.0/.

\section{References}

1. Statistik Austria. Österreichisches Krebsregister und Todesursachenstatistik. 2020. http://www.statistik.at/ web_de/statistiken/menschen_und_gesellschaft/gesund heit/krebserkrankungen/index.html. Accessed 14 Feb 2021.

2. Paireder M, Asari R, Radlspöck W, et al. Esophageal resection in Austria-preparing a national registry. Eur Surg. 2021;53(5):206-14. 
3. Arnold M, Soerjomataram I, Ferlay J, et al. Global incidence of oesophageal cancer by histological subtype in 2012. Gut. 2015;64(3):381-7.

4. Fatehi Hassanabad A, Chehade R, Breadner D, et al. Esophageal carcinoma: towards targeted therapies. Cell Oncol. 2020;43(2):195-209.

5. Bang A, Broomfield JA, Chan J, et al. Radiation dose mapping and anastomotic complications after trimodality therapy for esophageal cancers. Clin Transl Radiat Oncol. 2019;15:76-82.

6. Lordick F, Mariette C, Haustermans K, et al. Oesophageal cancer: ESMO Clinical Practice Guidelines for diagnosis, treatment and follow-up. Ann Oncol. 2016;27(suppl 5):v50-v7.

7. Mitchell DP, Yeluri S, Van der Wall H, et al. The real risk of nodal disease in $\mathrm{T} 1$ oesophageal adenocarcinoma. Eur Surg. 2020;52(3):110-7.

8. vanHagen P,HulshofMC, vanLanschotJJ, etal. Preoperative chemoradiotherapy for esophageal or junctional cancer. NEngl J Med. 2012;366(22):2074-84.

9. Low DE, Kuppusamy MK, Alderson D, et al. Benchmarking complications associated with esophagectomy. Ann Surg. 2019;269(2):291-8.

10. El-Sourani N, Kechagia C, Alfarawan F, et al. Classification and evaluation of anastomotic leaks after esophageal surgery-a tertiary university experience. Eur Surg. 2021; https:// doi.org/10.1007/s10353-021-00706-y.

11. Low DE, Alderson D, Cecconello I, et al. International consensus on standardization of data collection for complications associated with esophagectomy: Esophagectomy Complications Consensus Group (ECCG). Ann Surg. 2015;262(2):286-94.

12. Duan X, Bai W, Ma Z, et al. Management and outcomes of anastomotic leakage after McKeown esophagectomy: a retrospective analysis of 749 consecutive patients with esophageal cancer. Surg Oncol. 2020;34:304-9.

13. Fearon NM, Mohan HM, Fanning M, et al. Colonic interposition, a contemporary experience: technical aspects and outcomes. Updates Surg. 2021;73(5):1849-55.
14. MarksJL,HofstetterWL.Esophageal reconstruction withalternativeconduits. SurgClin NorthAm. 2012;92(5):1287-97.

15. Baker CR, Forshaw MJ, Gossage JA, et al. Long-term outcome and quality of life after supercharged jejunal interposition for oesophageal replacement. Surgeon. 2015;13(4):187-93.

16. Markar S, Gronnier C, Duhamel A, et al. Salvage surgery afterchemoradiotherapyin the management of esophageal cancer: Is it a viable therapeutic option? J Clin Oncol. 2015;33(33):3866-73.

17. Sokoya M, Vincent A, Cohn JE, et al. Comparison of radial forearm free flap and gastric pull-up in pharyngo-oesophageal reconstruction. Clin Otolaryngol. 2019;44(3):405-7.

18. Baek SM. Two new cutaneous free flaps: the medial and lateral thigh flaps. Plast Reconstr Surg. 1983;71(3):354-65.

19. Chen YB, Chen HC, Hahn LH. Major mandibular reconstruction with vascularized bone grafts: indications and selection of donor tissue. Microsurgery. 1994;15(4):227-37.

20. Weitgasser L, CotofanaS, Winkler M, et al. Detailed vascular anatomy of the medial femoral condyle and the significance of its use as a free flap. J Plast Reconstr Aesthet Surg. 2016;69(12):1683-9.

21. Forner D, Williams BA, Makki FM, et al. Late free flap failure in head and neck reconstruction: a systematic review. Ear Nose ThroatJ.2018;97(7):213-6.

22. Zhou W, Zhang WB, Yu Y, et al. Risk factors for free flap failure: a retrospective analysis of 881 free flaps for head and neck defect reconstruction. Int J Oral Maxillofac Surg. 2017;46(8):941-5.

Publisher's Note Springer Nature remains neutral with regard to jurisdictional claims in published maps and institutional affiliations. 